\section{Badgers guilty}

SIR - In his letter published on 11 December 1980 (page 532), Stephen Harris, among many other misleading and biased remarks, stated that the rate of decline in the incidence of tuberculosis (TB) in badgers in the South West was "paralleled" by a decline in the incidence of reactors in cattle herds not only in the South West but also in the rest of England. This statement was based on a graph which was attached to a copy of the letter sent to Lord Zuckerman and which is now reproduced (Fig.1). It was deduced from this that there might well be some common cause unconnected with the gassing campaign that was reducing the incidence of TB both in cattle and badgers throughout the country.

This "parallelism", however, was only secured by expressing all plotted values as percentages of the corresponding 1974 values. In Fig.2 I have plotted the breakdowns for the South West and for the rest of England on the same scale, subdividing the South West into (a) Gloucester, Avon and Wiltshire; $(b)$ Cornwall; and (c) Devon, Dorset and Somerset. The radical differences between the counties that have a high incidence of herd breakdowns, $(a)$ and $(b)$, and the rest of England $(d)$ is immediately apparent.

Table 10 of the report (Badgers, Cattle and Tuberculosis, Lord Zuckerman; HMSO, London, 1980) shows that the sources of infection are indeed different in the South West and in the rest of England. In groups $(a)$

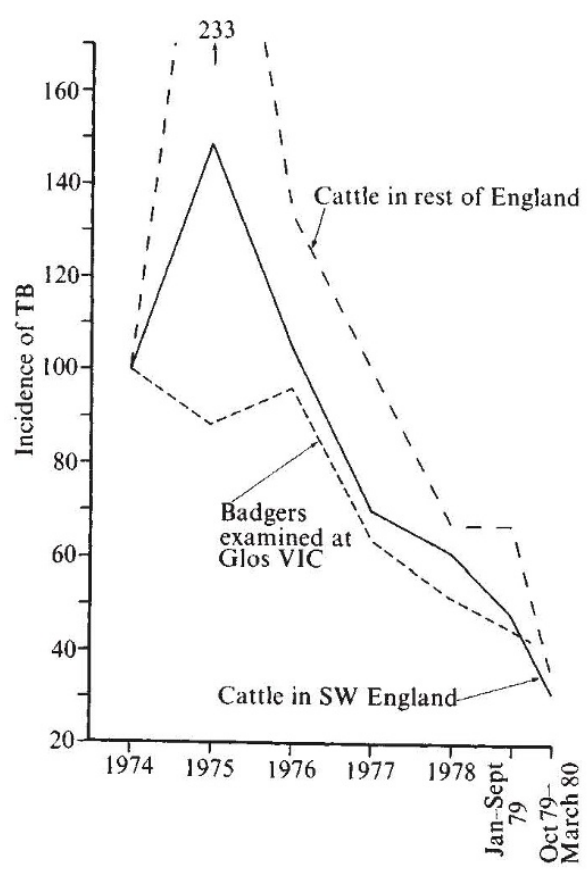

Fig.1 Annual changes in the incidence of TB in badgers sampled at the Gloucester Veterinary Investigation Centre (page 62 of the report) and herds of cattle in South-West England and herds of cattle in the rest of England (page 64). The first year for which data were available in the report from all three samples (1974) was taken as 100 per cent; for subsequent years, the level of TB was expressed as a percentage of the incidence in 1974. and $(c)$ three-quarters of the infections are attributed to badgers, but only 1 per cent to imported Irish cattle, whereas in the rest of Great Britain one half are attributed to imported Irish cattle and none to badgers.

In Cornwall (b), as Harris observes, only 15 per cent were definitely attributed to badgers, but as almost three-quarters of the causes of Cornish breakdown were recorded as "unknown"' and the remainder were either from purchased cattle or contiguous premises, it may be presumed that infection from badgers was much greater than this.

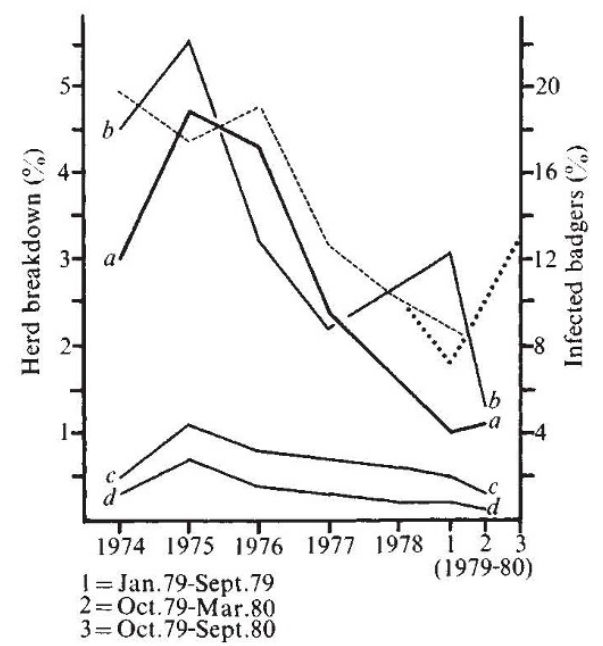

Fig.2 The percentages of herd breakdown are shown by full lines (from Table 19 of the report). The percentages of badgers found to be infected are shown by the broken line (Table 15). The dotted line gives some supplementary information from Table 18 (updated).

The fall in the incidence of herd breakdowns following the introduction of the gassing campaign in Gloucester/Avon agrees well with the reduction in the incidence of TB in badgers (broken line), bearing in mind that in addition to the reduction in the percentage incidence in badgers the numbers of badgers in close contact with herds must have been substantially reduced by the gassing.

The dotted line is derived from the latter part of Table 18, updated to September 1980, and indicates the rise in TB incidence in badgers following the cessation of gassing. (Updated figures for herd breakdowns in Gloucester/Avon and Wiltshire are not yet available).

The only support that Fig.2 gives to the suggestion that there was a common cause, other than appropriate action by ministry officials, affecting the decline in TB operating throughout the country is the substantial rise between 1974 and 1975. This cannot be related to infection from badgers. It may possibly have been due to climatic factors or to some improvement in the tuberculin test.

Figure 1 is a sad illustration of the way in which distorted and partial graphical presentation can be used for propaganda purposes.

FRANK YATES

Rothamsted Experimental Station

Harpenden, UK

\section{Rothschild retreat}

SIR - It is not clear from your leading article in the January $1 / 8$ issue (page 2) whether you regret the government's "retreat from Rothschild" or just the manner in which it is happening.

One of the difficulties for the Medical Research Council (MRC) at the time of the Rothschild report was the uncertainty as to the failures in the then current system which it was intended to correct. The Ministry of Health did not identify any omissions on the part of the MRC in their contribution to improving the health of the nation, nor did they subsequently propose contracts in fields of work outside the previous commitment of the MRC. Rothschild, in a thoughtless comment, referred to the very low expenditure on research into ageing, one of the fundamental aspects of all living things, but the understanding of which is nowhere in sight. He failed to ask the practical question as to how much of the MRC budget was spent on investigating diseases most common in old age. The answer would have been a major part.

There are, at present, three developments in medical research on which great hopes of practical gain are based: interferon, antibody production by hybridomas and DNA technology. How far the expectations will be justified remains to be seen, but the heavy investments of pharmaceutical companies throughout the world in these fields is evidence that the hopes are held widely. MRC laboratories were responsible for the first two discoveries and made a very big contribution to the third. None came from planned research but from "Dr Gowans' doctrine of investment in good ideas and good people".

It has been argued strongly that much more attention should be given to public health and preventive medicine and that the MRC's expenditure has the wrong emphasis. But one of the major advances in this field, the correlation of the incidence of lung cancer with cigarette smoking, came from work in an MRC unit.

No doubt governmental administration of civil science could be improved substantially, but the next enquiry which you appear to be advocating must surely start by identifying the omissions and failures of the present system whether in medical, agricultural or any other field of research before recommending changes to overcome them. Your generalized denigration of a system which has achieved obvious successes is not enough.

R.R. PORTER

Department of Biochemistry, University of Oxford, Oxford, UK

\section{Creating problems}

SIR - Your readers should know of the court action by creationists against the State of California, now expected to begin on 2 March. The suit, which is sponsored by the Creation Research Society of San Diego, is in the names of Segraves et al., students in California schools, including children of Mrs Nell Segraves of the society. The suit charges that the State of California has violated the

Continued on page 335 